I smoke (3)

I take fewer than 8 out of 10 prescribed doses of my regular preventer medication (3)

In the last month I have used my reliever inhaler more than once per day on average (3)

I sneeze, or get a runny, or a blocked nose when I do not have a cold (2)

In the last 5 years, I have attended a hospital emergency department or been admitted to hospital because of asthma (2)

I have a body mass index of $\geq 30$ (1)

I have not received asthma related education or a written asthma management plan (1)

I left school before sitting my A-levels/highers (1)

In summary, we systematically identified factors from the literature that are independently associated with the risk of asthma attack. These factors inform a questionnaire which will require validation and subsequent impact assessment.

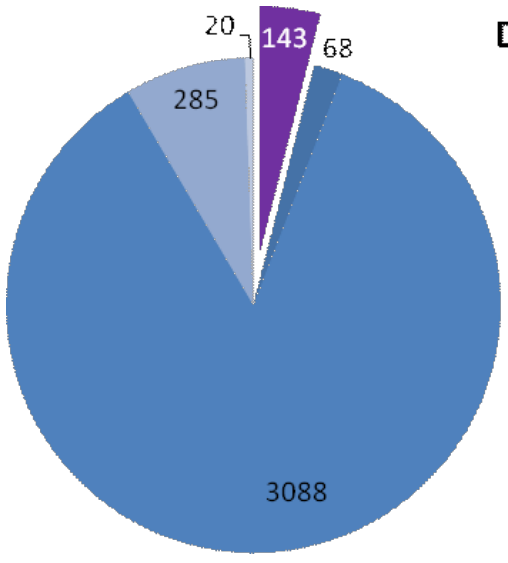

Distribution of Papers

- moluded in review

Duplicates from search

- Title screening exclusions

Abstract checklist exclusions

Full paper exclusions

Abstract S62 Figure 1

\section{S63 BASELINE ASTHMA CONTROL AND SEVERITY INFLUENCES THE OUTCOME OF VIRUS-INDUCED ASTHMA EXACERBATIONS}

doi:10.1136/thoraxjnl-2012-202678.069

'DJ Jackson, 'M Trujillo-Torralbo, 'J del-Rosario, 'J Footitt, 'P Mallia, ${ }^{2} \mathrm{OM}$ Kon, ${ }^{1} T$ Hansel, 'SL Johnston. 'National HeartLung Institute, Imperial College London, London, UK; 2Imperial College NHS Trust, London, UK

Background Rhinovirus (RV) infection is the most common cause of asthma exacerbations (AE). Mechanisms underlying this remain poorly understood. A human model of experimental RV induced AE has been developed however published studies have only recruited subjects with mild, well-controlled asthma naive to inhaled corticosteroid (ICS) therapy. The influence of asthma severity and baseline control on outcome remains unknown. For these studies to be more representative of those who experience virus-induced $\mathrm{AE}$ there is a need to establish the safety of using this model in subjects with moderate, poorly-controlled asthma and to investigate clinical outcomes. Method 48 adults - 14 healthy, 14 mild asthmatic, and 18 moderate asthmatic (defined by GINA) were recruited and inoculated nasally with RV-16. Daily symptom scores and spirometry were recorded throughout the study. Asthma control at baseline was recorded using the ACQ. Nasal lavage (NL) for viral shedding was performed on days $0,2,3,4,5,7,10$. Clinical infection was confirmed by demonstration of RV16 RNA by RT-PCR in NL and/or serum titre of RV-16 specific antibodies greater than $1: 4$ on $\mathrm{d} 42$.

Results $11 / 14$ healthy, $11 / 14$ mild asthmatic and 17/18 moderate asthmatic volunteers met criteria for infection. Both groups of asthmatics developed greater lower respiratory symptoms, falls in FEV1, and airway hyper-responsiveness (AHR) compared to healthy volunteers (all $P=<0.01)$. These changes were significantly greater in the moderate asthmatics than in the mild asthmatics $(P=<0.05)$. Poorly-controlled asthmatics experienced greater chest symptoms $(P=<0.01)$ and RV-induced falls in lung function $(P=<0.05) \mathrm{com}$ pared to subjects with well-controlled asthma.

Conclusion RV infection results in more severe chest symptoms and falls in lung function in moderate asthma than in mild asthma. Within the moderate group the poorly-controlled asthmatics experienced the most severe exacerbations. This occurred despite therapy with ICS. This is the first study to experimentally inoculate both moderate, poorly-controlled and milder well-controlled asthmatics. Both severity and baseline control appear to influence the outcome of virus-induced AE. Measures to improve control will significantly reduce the likelihood of a severe virus-induced $\mathrm{AE}$ and lessen the healthcare costs associated with them.

\section{S64 THE COST OF REFRACTORY ASTHMA IN THE UK - A PRELIMINARY ANALYSIS}

doi:10.1136/thoraxjnl-2012-202678.070

'S O'Neill, ${ }^{2} \mathrm{~J}$ Sweeney, ${ }^{3} \mathrm{C} 0$ Neill, ${ }^{4} \mathrm{C}$ Brightling, ${ }^{5} \mathrm{~A}$ Menzies-Gow, ${ }^{6} \mathrm{R}$ Niven, ${ }^{7} \mathrm{R}$ Chaudrai, ${ }^{8} \mathrm{C}$ Bucknall, ${ }^{9} \mathrm{~A}$ Mansur, ${ }^{10} \mathrm{C}$ Patterson, ${ }^{11} \mathrm{LG}$ Heaney on behalf of the British Thoracic Society Difficult Asthma Network. 'National University of Ireland, Galway. ${ }^{2}$ Centre for Infection Immunity, Queens University of Belfast; ${ }^{3}$ National University of Ireland, Galway; ${ }^{4}$ Institute for Lung Health, University of Leicester, Department of Infection, Inflammation Immunity, Leicester; ${ }^{5}$ Royal BromptonHarefield NHS Foundation Trust, London; ${ }^{6}$ The University of Manchester (MAHSC)University Hospital of South Manchester, Manchester; ' Gartnavel General Hospital, Glasgow, ${ }^{8}$ Glasgow Royal Infirmary, Glasgow; ${ }^{9} \mathrm{Heart}$ of England NHS Foundation Trust, Birmingham; ${ }^{10} \mathrm{Centre}$ for Public Health, Queens University of Belfast; "'Centre for Infection Immunity, Queens University of Belfast.

Introduction Refractory asthma poses a potentially significant burden in terms of healthcare costs. Relatively little is known about the cost of treatment or what factors explain variations in treatment costs. This study uses data from the British Thoracic Society (BTS) Difficult Asthma Registry to estimate the healthcare costs associated with a sample of well-characterised refractory asthmatics and examines the role of a range of factors in explaining variations in healthcare.

Methods In this analysis data were extracted from the Registry on 689 patients and examined healthcare utilisation including all prescribed medicines, hospital inpatient stays, ITU stays, A\&E/GP visits and CT scans over a 12 month period prior to the patient first being assessed at the specialist clinics. Patient characteristics included age, gender, lung function, clinical centre were care was provided, adherence status, BMI and whether or not the patient was on maintenance oral steroids. Unit costs were based on standard published sources. Costs were examined by category with respect to patient characteristics and total cost with respect to patient characteristics in multivariate regression analyses.

Results Mean total treatment cost among refractory asthmatics ranged between $£ 3,402(\mathrm{SD}=2,680)$ to $£ 4,234$ ( $\mathrm{SD}=£ 3,036)$. In a comparator non-refractory group mean total cost ranged from $£ 1,944(\mathrm{SD}=£ 1,728)$ and $£ 2,565$ ( $£ 2,065)$. Drug costs comprised approximately $58 \%$ of all costs in refractory asthmatics and approximately $55 \%$ in the non-refractory group. In the refractory group significant predictors of total costs were FEV1, clinical centre in which care was provided, maintenance oral steroids and BMI. Patients who were on maintenance steroids cost $48 \%$ more than those who were not. Patients who were morbidly obese cost approximately 23\% more than those who were normal weight.

Conclusion Treating individuals with refractory asthma presents a significant cost to the health service. The role of maintenance steroids in cost is dramatic and may relate to the impact of steroid induced morbidity and warrants further investigation. 